\title{
A general norbornyl based synthetic approach to carbasugars and 'confused' carbasugars
}

\author{
Goverdhan Mehta,* Pinaki Talukdar and Narinder Mohal \\ Department of Organic Chemistry, Indian Institute of Science, Bangalore 560 012, India
}

\begin{abstract}
The norbornyl system has been recognized simply as a 'locked' carbasugar and a short, general approach to carbasugars and their new siblings, 'confused' carbasugars, from readily available 7-ketonorbornanes is reported.
\end{abstract}

In the pursuit of scientific research, sometimes the most obvious is either taken for granted or escapes attention. The bicyclo[2.2.1] heptane (norbornyl) system has been explored by generations of organic chemists in a myriad ways ranging from mechanistic and stereochemical probes to starting point for diverse syntheses. ${ }^{1}$ However, the $\mathrm{C}_{7}$-framework of the norbornyl system has not been recognized as a simple 'locked' carbasugar from which the six-membered $\mathrm{C}_{7}$-carbasugar skeleton can be easily retrieved through 'unlocking' involving $\mathrm{C} 1-\mathrm{C} 7$ or $\mathrm{C} 4-\mathrm{C} 7$ bond scission. Considering the current widespread interest in the synthesis of carbasugars, ${ }^{2}$ we regard our norbornyl approach as short, simple and conceptually different. We demonstrate here that indeed, a simple 7-norbornenone derivative like 1 can be readily elaborated to a variety of carbasugars $\mathbf{2}$ and to related new entities that we name 'confused' carbasugars 3. The 'confused' carbasugars 3 have the same level of oxygenation on the cyclohexanoid framework as the carbasugars, but the hydroxymethyl and the 'para' hydroxy groups are interchanged (see bold portions in $\mathbf{2}$ and $\mathbf{3}$ ). In view of the well-established importance of carbasugars $\mathbf{2}$ and its congeners in glycomimicry, the first time access to their new siblings, the 'confused' carbasugars $\mathbf{3}$, should stimulate interest in the evaluation of their biological activity profile.
endo-2-Acetoxy-7-norbornenone $\mathbf{5}$ and its precursor ketal 4, readily available through Diels-Alder reaction between 5,5-dimethoxy-1,2,3,4-tetrachlorocyclopentadiene and vinyl acetate, followed by reductive dehalogenation, ${ }^{2 b, 3}$ were chosen as the starting points of our projected syntheses. ${ }^{4}$ We recognized that dihydroxylation of the norbornene double bond and cleavage of the C1-C7 bond with amplification of functionality would provide direct access to carbasugars. Consequently, 4 was subjected to $\mathrm{OsO}_{4}$-mediated dihydroxylation exclusively from the exo-face to furnish $\mathbf{6}$. A one-pot cis-diol protection and acetal deprotection in $\mathbf{6}$ delivered the keto-acetonide 7 (Scheme 1). Baeyer-Villiger oxidation of 7 led to a regioisomeric mixture of lactones 8 and $\mathbf{9}$ (13:87). $\mathrm{LiAlH}_{4}$ reduction of $\mathbf{8}$ and 9 yielded triols $\mathbf{1 0}$ and 11, respectively. Acetonide deprotection in $\mathbf{1 0}$ led to the pseudo- $\alpha$-DL-talopyranose carbasugar 12a (characterized as the pentaacetate $\mathbf{1 2}^{4 \mathbf{4 a , 5}}$ ). The same deprotection in $\mathbf{1 1}$ delivered the 'confused' carbasugar 13a (characterized as the pentaacetate $\mathbf{1 3 b}^{6}$ ) quite easily (Scheme 1).

To introduce stereochemical diversity in our synthetic approach and to fine-tune the relative ratios of the carba- and 'confused' carbasugar formation, an alternate strategy was executed. Chemoselective Baeyer-Vil-<smiles>OCC1CC(O)C(O)C(O)C1O</smiles>

Carbasugar

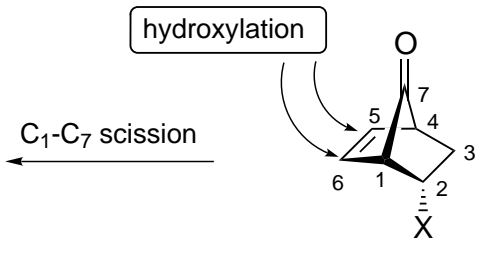

$1 \mathrm{X}=\mathrm{OH}$, OAc etc.<smiles>OCC1C(O)C(O)C(O)C(O)C1O</smiles>

3

'Confused' Carbasugar

\footnotetext{
* Corresponding author.
} 

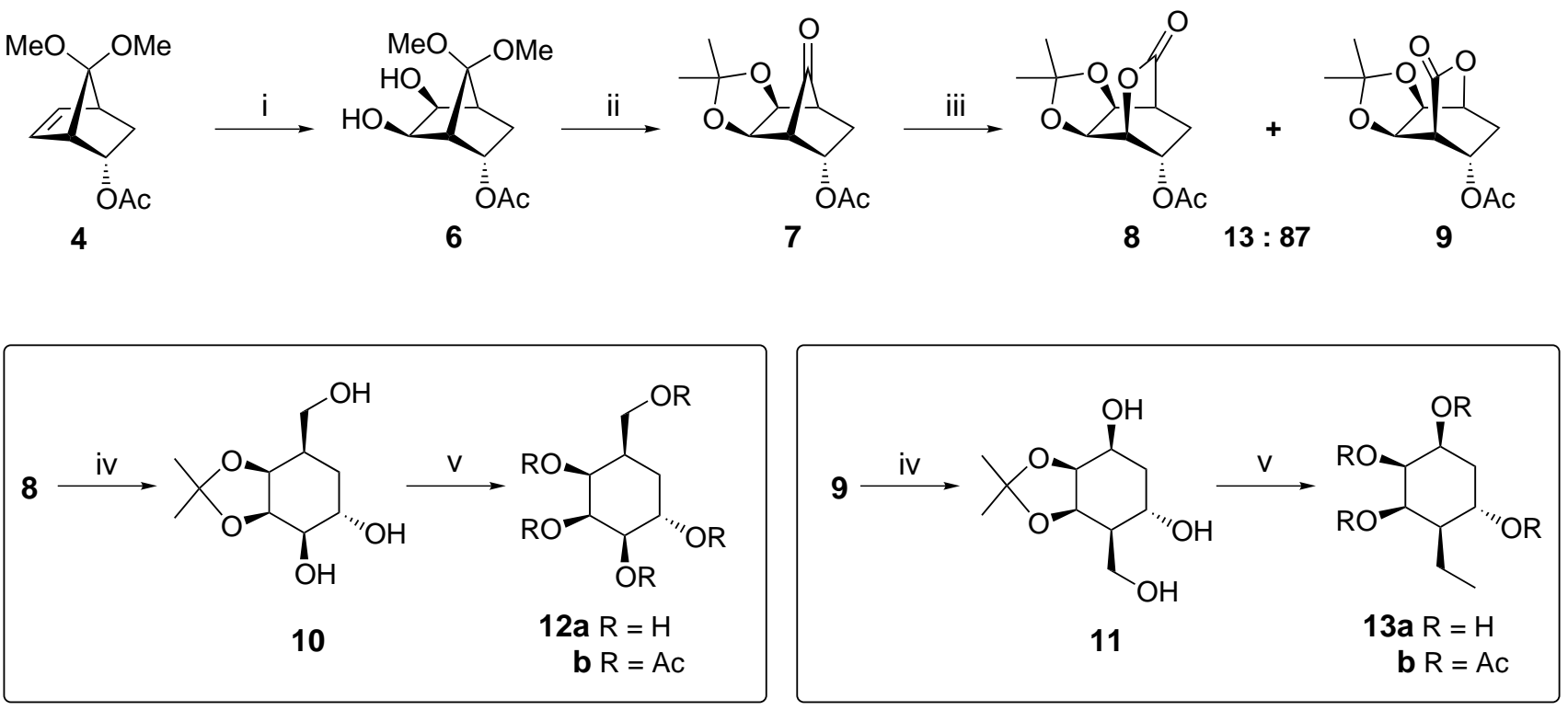

Scheme 1. Reagents and conditions: (i) $\mathrm{OsO}_{4}, \mathrm{NMMO}\left(50 \%\right.$ aq. solution, 4 equiv.), $\mathrm{Me}_{2} \mathrm{CO}-\mathrm{H}_{2} \mathrm{O}(4: 1)$, rt, 30 h, 80\%; (ii) Amberlyst-15, $\mathrm{Me}_{2} \mathrm{CO}$, rt, overnight, 70\%; (iii) $\mathrm{MCPBA}^{\mathrm{NaHCO}}, \mathrm{DCM}, 0-5^{\circ} \mathrm{C}, 3-4 \mathrm{~h}$, quant.; (iv) $\mathrm{LAH}, \mathrm{THF}, 0-5^{\circ} \mathrm{C}, 2-3 \mathrm{~h}$, 70\%; (v) (a) Amberlyst-15, aq. $\mathrm{MeOH}$, rt, overnight, (b) $\mathrm{Ac}_{2} \mathrm{O}, \mathrm{Py}, \mathrm{rt}$, overnight, 72\% (two steps).

liger oxidation of norbornenone $\mathbf{5}$ furnished the lactones 14 and 15 (30:70) (Scheme 2). The minor lactone 14 was first subjected to $\mathrm{LiAlH}_{4}$ reduction to furnish cyclohexene triol 16. $\mathrm{OsO}_{4}$-mediated dihydroxylation on 16 was stereospecific from the face opposite to the allylic hydroxyl group and furnished pseudo- $\alpha$-DLaltropyranose carbasugar $\mathbf{1 7 a}$ and was fully characterized as its pentaacetate $\mathbf{1 7 b} .^{7}$ In an alternate sequence, 16 was subjected to epoxidation with $m$-chloroperbenzoic acid in aqueous medium to furnish trans-epoxide 18 in a stereoselective manner. Interestingly, the epoxidation of $\mathbf{1 6}$ was dictated by steric considerations rather than by the usually encountered directing influence of the allylic hydroxyl group. ${ }^{8,9}$ Acid-catalyzed opening of the epoxide ring in $\mathbf{1 8}$ was regioselective and pseudo- $\alpha-$

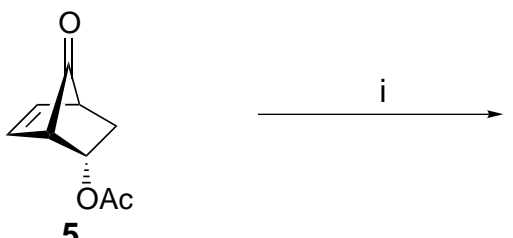

DL-mannopyranose 19a (characterized as the pentaacetate $\mathbf{1 9} \mathbf{b}^{5,10}$ ) was the main product formed with only traces of the regioisomeric pseudo- $\alpha$-DL-idopyranose. ${ }^{11}$ In an analogous manner, the major lactone $\mathbf{1 5}$ from $\mathbf{5}$ was reduced with $\mathrm{LiAlH}_{4}$ to furnish two cyclohexene triols 20 and 21 (75:25) (Scheme 3). Quite unexpectedly, epimerization had occurred during the hydride reduction of 15, possibly at the intermediate aldehyde stage and the hydroxymethyl group in $\mathbf{2 1}$ was cis to the neighbouring hydroxyl group. Dihydroxylation of 20 with $\mathrm{OsO}_{4}$ proceeded stereoselectively to furnish the 'confused' carbasugar 22a (characterized as pentaacetate $\mathbf{2 2} \mathbf{b}^{6}$ ). Epoxidation of $\mathbf{2 0}$ took a course analogous to $\mathbf{1 6}$ and furnished the trans-epoxide 23. ${ }^{8,9}$ Acid-catalyzed opening in $\mathbf{2 3}$ led to a new 'confused' carbasugar

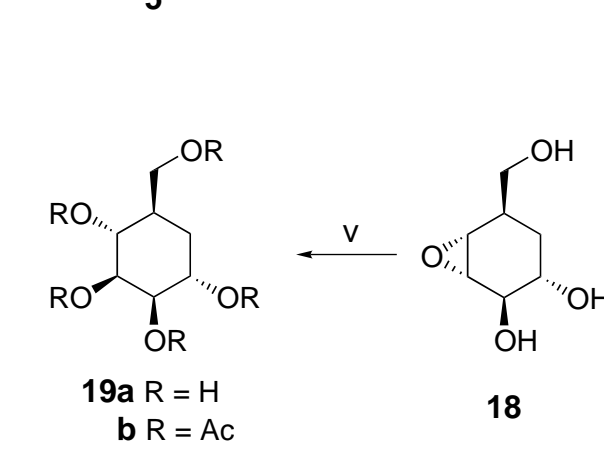<smiles>CC(=O)O[C@H]1C[C@@H]2C=C[C@H]1OC2=O</smiles>

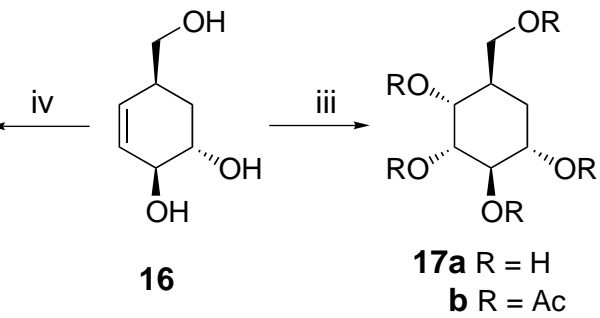

Scheme 2. Reagents and conditions: (i) MCPBA, $\mathrm{Na}_{2} \mathrm{CO}_{3}, \mathrm{DCM}, 0^{\circ} \mathrm{C}-\mathrm{rt}, 4-5 \mathrm{~h}, 94 \%$; (ii) LAH, THF, $-15^{\circ} \mathrm{C}, 2 \mathrm{~h}, 70 \%$; (iii) (a) $\mathrm{OsO}_{4}, \mathrm{NMMO}\left(50 \%\right.$ aq. solution, 4 equiv.), $\mathrm{Me}_{2} \mathrm{CO}-\mathrm{H}_{2} \mathrm{O}$ (4:1), rt, overnight, (b) $\mathrm{Ac}_{2} \mathrm{O}, \mathrm{Py}, \mathrm{rt}, 36 \mathrm{~h}, 78 \%$ (two steps); (iv) MCPBA, $\mathrm{H}_{2} \mathrm{O}, \mathrm{rt}, 2$ days, 75\%; (v) (a) cat. $\mathrm{HClO}_{4}(70 \%), \mathrm{H}_{2} \mathrm{O}, \mathrm{rt}, 24 \mathrm{~h}$, (b) $\mathrm{Ac}_{2} \mathrm{O}, \mathrm{Py}, \mathrm{rt}, 35 \mathrm{~h}, 73 \%$ (two steps). 


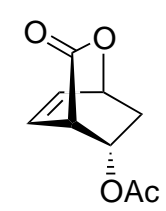

15<smiles>[2H]C1CC([2H])C(CO)C([2H])C1[2H]</smiles>

22a $\mathrm{R}=\mathrm{H}$

b $R=A c$

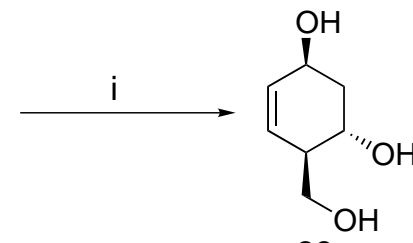

20

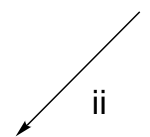

ii<smiles>OC[C@H]1[C@@H](O)C[C@@H](O)[C@@H]2O[C@H]12</smiles>

23<smiles>OCC1C=CC(O)CC1O</smiles>

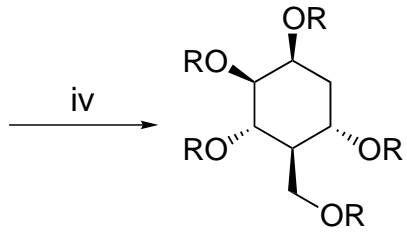

24a $\mathrm{R}=\mathrm{H}$

b $R=A c$

Scheme 3. Reagents and conditions: (i). LAH, THF, $-15^{\circ} \mathrm{C}, 2 \mathrm{~h}, 70 \%$; (ii) (a) $\mathrm{OsO}_{4}$, NMMO (50\% aq. solution, 4 equiv.), $\mathrm{Me}_{2} \mathrm{CO}-\mathrm{H}_{2} \mathrm{O}$ (4:1), rt, overnight, (b) $\mathrm{Ac}_{2} \mathrm{O}, \mathrm{Py}, \mathrm{rt}, 36$ h, 76\% (two steps); (iii) $\mathrm{MCPBA}_{2} \mathrm{H}_{2} \mathrm{O}, \mathrm{rt}, 2$ days, 77\%; (iv) (a) cat. $\mathrm{HClO}{ }_{4}$ $(70 \%), \mathrm{H}_{2} \mathrm{O}, \mathrm{rt}, 24 \mathrm{~h}$, (b) $\mathrm{Ac}_{2} \mathrm{O}, \mathrm{Py}, \mathrm{rt}, 40 \mathrm{~h}, 72 \%$ (two steps).

24a (characterized as pentaacetate $\mathbf{2 4} \mathbf{b}^{6}$ ) in a regioselective manner (Scheme 3). Similar transformations, dihydroxylation and epoxidation ring opening could also be executed on 21, thus providing additional diversity among the 'confused' carbasugar family. We have carried out preliminary studies of the glycosidase inhibition ability of 'confused' carbasugars 13a, 22a and 24a towards a panel of six glycosidases. However, no significant inhibition has been observed.

In summary, we have delineated a new and exceptionally simple approach to carbasugars and their siblings the 'confused' carbasugars, which has built-in flexibility to create stereochemical diversity. The 'confused' carbasugars being new entities, deserved to be evaluated further and elaborated to their amino derivatives as well as to oligomers. Efforts along these lines are in progress.

\section{Acknowledgements}

We thank Dr. M. Vairamani, IICT, Hyderabad for the HRMS data and the SIF facility at IISc for the high field NMR spectra. One of us (N.M.) thanks CSIR for a research fellowship. Part of this was carried out at the University of Hyderabad.

\section{References}

1. Norbornyl route to prostaglandins is a seminal example. For some leading references, see: (a) Corey, E. J.; Cheng, Xue-Min. The Logic of Chemical Synthesis, Wiley-Interscience: New York, 1991; (b) Paquette, L. A.; Learn, K. S.; Romine, J. L.; Lin, H.-S. J. Am. Chem. Soc. 1988, 110, 879; (c) Sgarbi, P. W. M.; Clive, D. L. J. Chem.
Commun. 1997, 2157; (d) Mehta, G.; Khan, F. A. J. Am. Chem. Soc. 1990, 112, 6140; (e) Mehta, G.; Chandrasekhar, J. Chem. Rev. 1999, 99, 1437.

2. (a) Suami, T. Top. Curr. Chem. 1990, 154, 257; (b) Ferrier, R. J. Chem. Rev. 1993, 93, 2779; (c) Hudlicky, T.; Entwistle, D. A.; Pitzer, K. K.; Thorpe, A. J. Chem. Rev. 1996, 96, 1195; (d) Landais, Y. Chimia 1998, 52, 104.

3. (a) Jung, M. E.; Hudspeth, J. P. J. Am. Chem. Soc. 1977, 99, 5508; (b) Mehta, G.; Mohal, N. Tetrahedron Lett. 1999, 40, 5791.

4. For norbornyl based approach to cyclohexitols emanating from our laboratory, see: (a) Mehta, G.; Mohal, N.; Lakshminath, S. Tetrahedron Lett. 2000, 41, 3505; (b) Mehta, G.; Lakshminath, S. Tetrahedron Lett. 2000, 41, 3509.

5. Pingli, P.; Vandewalle, M. Synlett 1994, 228.

6. All compounds reported here were fully characterized on the basis of their spectral (IR, ${ }^{1} \mathrm{H},{ }^{13} \mathrm{C}$ NMR, MS) and analytical data. Selected spectral data. 13b: $\mathrm{Mp}=116$ $117^{\circ} \mathrm{C}$; IR (neat) $v_{\max } 1746 \mathrm{~cm}^{-1} ;{ }^{1} \mathrm{H}$ NMR $(300 \mathrm{MHz}$; $\left.\mathrm{CDCl}_{3}\right) \delta 5.42-5.41(\mathrm{~m}, 1 \mathrm{H}), 5.28-5.13(\mathrm{~m}, 3 \mathrm{H}), 4.26(\mathrm{dd}$, $1 \mathrm{H}, J=11.5,6.0 \mathrm{~Hz}), 4.15(\mathrm{dd}, 1 \mathrm{H}, J=11.5,8.1 \mathrm{~Hz})$, 2.42-2.34 (m, 1H), 2.30-2.15 (m, 1H), 2.08 (s, 9H), 2.06 $(\mathrm{s}, 3 \mathrm{H}), 2.05(\mathrm{~s}, 3 \mathrm{H}), 1.86-1.81(\mathrm{~m}, 1 \mathrm{H}) ;{ }^{13} \mathrm{C}$ NMR $(50$ $\left.\mathrm{MHz} ; \mathrm{CDCl}_{3}\right) \delta 170.93,170.00,169.90,169.85,169.70$, $69.57,67.49,67.28,67.12,60.79,41.82,29.43,21.15$, 20.92, 20.86, 20.82, 20.75; MS (EI, $70 \mathrm{eV}) \mathrm{m} / z 388\left(\mathrm{M}^{+}\right.$, $<1 \%$ ), 328 (5), 166 (90), 124 (70), 83 (74), 43 (100); anal. found: $\mathrm{C}, 52.49 ; \mathrm{H}, 6.26 . \mathrm{C}_{17} \mathrm{H}_{24} \mathrm{O}_{10}$ requires $\mathrm{C}, 52.57 ; \mathrm{H}$, 6.23. 22b: $\mathrm{Mp}=102-103^{\circ} \mathrm{C}$; IR (thin film) $v_{\max } 1744 \mathrm{~cm}^{-1}$; ${ }^{1} \mathrm{H}$ NMR (300 MHz; $\left.\mathrm{CDCl}_{3}\right) \delta 5.31-5.27(\mathrm{~m}, 1 \mathrm{H}), 5.17$ (dd, 1H, $J=11.3,2.7 \mathrm{~Hz}), 5.13-5.03(\mathrm{~m}, 2 \mathrm{H}), 4.19$ (dd, $1 \mathrm{H}, J=11.7,2.1 \mathrm{~Hz}), 4.12(\mathrm{dd}, 1 \mathrm{H}, J=11.7,2.7 \mathrm{~Hz})$, 2.36-2.17 (m, 1H), $2.23(\mathrm{~m}, 1 \mathrm{H}), 2.15(\mathrm{~s}, 3 \mathrm{H}), 2.13(\mathrm{~s}$, $3 \mathrm{H}), 2.10(\mathrm{~s}, 3 \mathrm{H}), 2.06(\mathrm{~s}, 3 \mathrm{H}), 2.02(\mathrm{~s}, 3 \mathrm{H}), 1.89-1.80(\mathrm{~m}$, $1 \mathrm{H}) ;{ }^{13} \mathrm{C}$ NMR $\left(75 \mathrm{MHz} ; \mathrm{CDCl}_{3}\right) \delta 170.91,169.91$, $169.79,169.39,169.27,68.10,67.40,67.09,66.29,58.18$, 40.34, 30.67, 21.04, 20.99, 20.83, 20.77, 20.69; MS (EI, 70 eV) $m / z 388\left(\mathrm{M}^{+},<1 \%\right), 328$ (20), 166 (44), 124 (66), 43 
(100); anal. found: $\mathrm{C}, 52.61 ; \mathrm{H}, 6.25 . \mathrm{C}_{17} \mathrm{H}_{24} \mathrm{O}_{10}$ requires C, 52.57; H, 6.23. 24b: $\mathrm{Mp}=108-110^{\circ} \mathrm{C} ; v_{\max }$ (thin film) $1745 \mathrm{~cm}^{-1} ;{ }^{1} \mathrm{H}$ NMR $\left(300 \mathrm{MHz} ; \mathrm{CDCl}_{3}\right) \delta 5.48-5.38(\mathrm{~m}$, $2 \mathrm{H}), 5.14(\mathrm{dt}, 1 \mathrm{H}, J=11.4,4.5 \mathrm{~Hz}), 4.93(\mathrm{dd}, 1 \mathrm{H}, J=9.9$, $3.3 \mathrm{~Hz}), 4.09$ (d, $2 \mathrm{H}, J=3.0 \mathrm{~Hz}), 2.34(\mathrm{td}, 1 \mathrm{H}, J=13.8$, $4.5 \mathrm{~Hz}), 2.16$ (s, 3H), $2.08(\mathrm{~s}, 3 \mathrm{H}), 2.07$ (s, 3H), 2.07-2.05 (m, 1H), 2.05 (s, 3H), $1.99(\mathrm{~s}, 3 \mathrm{H}), 1.73-1.63(\mathrm{~m}, 1 \mathrm{H}) ;{ }^{13} \mathrm{C}$ NMR $\left(75 \mathrm{MHz} ; \mathrm{CDCl}_{3}\right) \delta 170.82,170.20,169.99,169.87$, 169.62, 73.09, 67.28, 67.05, 65.97, 58.25, 44.95, 33.21, 20.99, 20.92, 20.69, 20.61 (2C); MS (EI, $70 \mathrm{eV}) \mathrm{m} / z 388$ $\left(\mathrm{M}^{+}\right)$, 328, 166, 124, 95, 43; anal. found: C, 52.74; $\mathrm{H}$, 6.26. $\mathrm{C}_{17} \mathrm{H}_{24} \mathrm{O}_{10}$ requires $\mathrm{C}, 52.57 ; \mathrm{H}, 6.23$.
7. Suami, T.; Ogawa, S.; Ishibashi, T.; Kasahara, I. Bull. Chem. Soc. Jpn. 1976, 49, 1388.

8. (a) Henbest, H. B.; Wilson, R. A. L. J. Chem. Soc. 1957, 1958; (b) Adam, W.; Wirth, T. Acc. Chem. Res. 1999, 32, 703.

9. The trihydroxy-olefins $\mathbf{1 6}$ and $\mathbf{2 0}$ and their derived triacetates undergo epoxidation from the same face, indicating that these reactions are sterically controlled and hydroxy groups in $\mathbf{1 6}$ and $\mathbf{2 0}$ have no directing role.

10. Takahashi, T.; Kotsubo, H.; Iyobe, A.; Namiki, T.; Koizumi, T. J. Chem. Soc., Perkin Trans. 1 1990, 3065.

11. Paulsen, H.; von Deyn, W. Liebigs Ann. Chem. 1987, 125. 